\title{
Simplices and Spectra of Graphs
}

\author{
Bojan Mohar · Igor Rivin
}

Received: 8 September 2008 / Revised: 24 May 2009 / Accepted: 10 June 2009 /

Published online: 7 July 2009

(C) Springer Science+Business Media, LLC 2009

\begin{abstract}
In this note we show that the $(n-2)$-dimensional volumes of codimension 2 faces of an $n$-dimensional simplex are algebraically independent quantities of the volumes of its edge-lengths. The proof involves computation of the eigenvalues of Kneser graphs. We also show examples of families of simplices (of dimension 4 or greater) which show that the set of $(n-2)$-dimensional volumes of $(n-2)$ dimensional faces of a simplex do not determine its volume.
\end{abstract}

Keywords Simplex · Uniqueness · Graph spectrum · Representation theory · Kneser graph · Face volumes · Quantum gravity

\section{Introduction}

Let $\mathcal{T}_{n}$ be the set of congruence classes of $n$-simplices in Euclidean space $\mathbb{E}^{n}$. The set $\mathcal{T}_{n}$ is an open manifold (also a semi-algebraic set) of dimension $\left(\begin{array}{c}n+1 \\ 2\end{array}\right)$. Coincidentally,

\footnotetext{
Mohar was supported in part by the ARRS Research Program P1-0297, by an NSERC Discovery Grant and by the Canada Research Chair program. He was on leave from Department of Mathematics, IMFM \& FMF, University of Ljubljana, Ljubljana, Slovenia.

Igor Rivin was supported in part by the National Science Foundation. He would like to thank the American Institute of Mathematics for an invitation to the workshop on "Rigidity and Polyhedral Combinatorics", where this work was started. Igor Rivin has benefited from discussions with Bob Connelly, Ezra Miller, and Igor Pak.
}

A previous version of this work had appeared in [10].

B. Mohar

Department of Mathematics, Simon Fraser University, Burnaby, British Columbia V5A 1S6, Canada e-mail:mohar@sfu.ca

I. Rivin ( $₫)$

Department of Mathematics, Temple University, Philadelphia, USA

e-mail: rivin@temple.edu 
a simplex $T \in \mathcal{T}_{n}$ is determined by the $\left(\begin{array}{c}n+1 \\ 2\end{array}\right)$ lengths of its edges. Furthermore, the square of the volume of $T \in \mathcal{T}_{n}$ is a polynomial in the squares of the edge-lengths $\ell_{i j}=\left\|v_{i}-v_{j}\right\|_{2}(1 \leq i<j \leq n+1)$, where $v_{1}, \ldots, v_{n+1}$ are the vertices of $T$. This polynomial is given by the Cayley-Menger determinant formula (cf., e.g., [5] or [2]):

$$
V^{2}(T)=\frac{(-1)^{n+1}}{2^{n}(n !)^{2}} \operatorname{det} C,
$$

where $C$ is the Cayley-Menger matrix of dimension $(n+2) \times(n+2)$ whose rows and columns are indexed by $\{0,1, \ldots, n+1\}$ and whose entries are defined as follows:

$$
C_{i j}= \begin{cases}0, & i=j, \\ 1, & \text { if } i=0 \text { or } j=0, \text { and } i \neq j, \\ \ell_{i j}^{2}, & \text { otherwise. }\end{cases}
$$

Note that an $n$-simplex has $\left(\begin{array}{c}n+1 \\ 2\end{array}\right)$ edges and the same number of $(n-2)$ dimensional faces, and so the following question is natural:

Question 1 Is the congruence class of every $n$-simplex determined by the $(n-2)$ dimensional volumes of its $(n-2)$-faces?

Question 1 must be classical, but the earliest reference stating it that we are aware of is Warren Smith's Ph.D. thesis [11].

At the AIM workshop on Rigidity and Polyhedral Combinatorics, Bob Connelly (who was unaware of [11]) raised the following:

Question 2 Is the volume of every $n$-simplex determined by the $(n-2)$-dimensional volumes of its $(n-2)$-faces?

In fact, Connelly stated Question 2 for $n=4$, which is the first case where the question is open. For $n=3$ the answer is trivially "Yes", since $3-2=1$ and we are simply asking if the volume of the simplex is determined by its edge-lengths. In dimension 2, the answer is trivially "No", since $2-2=0$ and the volume of codimension- 2 faces of a triangle carries no information. Connelly further wondered whether the volume of a 4-simplex is integral over the ring generated by the areas of the two-dimensional faces.

Clearly, the affirmative answer to Question 1 would imply an affirmative answer to Question 2. In this paper, we first show that the answer to Question 2, and hence also to Question 1, is negative for every $n \geq 4$. We actually found out that this has been answered previously for $n=4$ in [1], where an example is given and attributed to Philip Tuckey; see also [3]. The referee has pointed out that a set of counterexamples for every $n \geq 4$ (different from ours) was given by P. McMullen in [9, Sect. 5].

Our examples are given in a separate section. Several reasons suggest that the following question may still have an affirmative answer:

Question 3 Is it true that, for every choice of $\left(\begin{array}{c}n+1 \\ 2\end{array}\right)$ positive real numbers, there are only finitely many congruence classes of $n$-simplices whose $(n-2)$-dimensional volumes of the $(n-2)$-faces are equal to these numbers? 
In this note we show that a weaker statement holds:

Theorem 4 The $\left(\begin{array}{c}n+1 \\ 2\end{array}\right)(n-2)$-dimensional volumes of the $(n-2)$-faces of an $n$-simplex are algebraically independent over $\mathbb{C}\left[\ell_{i j} ; 1 \leq i<j \leq n+1\right]$.

Theorem 4 is clearly a necessary step in the direction of resolving Question 3, but is far from sufficient. To show it, consider the map of $\mathbb{R}^{(n+1) n / 2}$ to $\mathbb{R}^{(n+1) n / 2}$, which sends the vector $\ell$ of edge-lengths of an $n$-simplex to the vector $Y$ of volumes of $(n-2)$-dimensional faces. To show Theorem 4 , it is enough to check that the Jacobian $J(\ell)=\partial Y / \partial \ell$ is non-singular at one point. We will use the most obvious point $p_{1}$, the one corresponding to a regular simplex with all edge-lengths equal to 1 . By symmetry considerations, the Jacobian $J\left(p_{1}\right)$ can be written as $J\left(p_{1}\right)=c M$, where $c$ is a constant and $M$ is

$$
M_{e, F}= \begin{cases}1, & \text { if the edge } e \text { is incident with the }(n-2) \text {-face } F, \\ 0, & \text { otherwise. }\end{cases}
$$

The first observation is that the constant $c$ above is not equal to 0 :

\section{Lemma 5}

$$
J\left(p_{1}\right)=\frac{1}{(n-2) !(n-1)^{1 / 2} 2^{(n-4) / 2}} M .
$$

Proof Let $v=\frac{(n-1)^{1 / 2}}{(n-2) ! 2^{(n-2) / 2}}$ denote the $(n-2)$-dimensional volume of the regular $(n-2)$-simplex with all edge-lengths 1 . Let us observe that the volume of a $k$-dimensional simplex is a homogeneous function of degree $k$ of the edge-lengths. An application of Euler's Homogeneous Function Theorem shows that at $p_{1}$,

$$
\frac{\partial Y_{F}}{\partial \ell_{e}}= \begin{cases}\frac{2}{n-1} v, & \text { if the edge } e \text { is incident with the }(n-2) \text {-face } F \\ 0, & \text { otherwise. }\end{cases}
$$

This implies that $c=\frac{2}{n-1} v$ and completes the proof.

\section{The Eigenvalues of $M$}

As shown above, Theorem 4 reduces to the assertion that the determinant of the matrix $M$ is not zero. We will actually be able to compute all eigenvalues of $M$, which is of interest in its own right.

Theorem 6 The eigenvalues of $M$ are $\lambda_{1}=\left(\begin{array}{c}n-1 \\ 2\end{array}\right)$ (simple eigenvalue), $\lambda_{2}=1$ with multiplicity $\frac{1}{2}(n+1)(n-2)$, and $\lambda_{3}=2-n$ with multiplicity $n$.

Corollary 7 The absolute value of the determinant of $M$ equals

$$
\frac{1}{2}(n-2)^{n+1}(n-1) \neq 0,
$$

for $n>2$. 
To prove Theorem 6, let us first observe that the $\left(\begin{array}{c}n+1 \\ 2\end{array}\right)$ rows of $M$ are indexed by the 2-element subsets of the set $R=\{1, \ldots, n+1\}$, and its columns are indexed by the $(n-1)$-subsets $F$ of $R$. By replacing each column index $F$ with its complement $R \backslash F$, the columns are indexed by the same set as the rows. After this convention, the matrix $M$ becomes a symmetric matrix with zero diagonal since $M_{e, f}=1$ if and only if $e \subseteq R \backslash f$, which is equivalent to $f \subseteq R \backslash e$. Therefore, $M$ is the adjacency matrix of a graph $G_{n}$ whose vertices are the 2-element subsets of $R$, and two of them are adjacent if and only if they are disjoint. Thus, the complement $\bar{G}_{n}$ of $G_{n}$ is isomorphic to the line graph $L\left(K_{n+1}\right)$ of the complete graph $K_{n+1}$ on $n+1$ vertices.

The eigenvalues of $L\left(K_{n+1}\right)$ are (see [4, p. 19]): $t_{1}=2 n-2, t_{2}=-2$, and $t_{3}=n-3$, with the same multiplicities (respectively) as claimed above for the eigenvalues of $M$. Since the graph $L\left(K_{n+1}\right)$ is regular, it is an easy exercise to see that its adjacency matrix $A$ and the adjacency matrix $M$ of its complement have the same set of eigenvectors. By using the fact that $A+M+I=x^{\mathrm{t}} \cdot x$, where $x=(1, \ldots, 1)^{\mathrm{t}}$ is the eigenvector of $A$ and $M$ corresponding to the dominant eigenvalues of these matrices, we conclude that the eigenvalues of $M$ are $\lambda_{1}=\left(\begin{array}{c}n+1 \\ 2\end{array}\right)-t_{1}-1$ and $\lambda_{i}=-t_{i}-1$ for $i=2,3$ (preserving multiplicities). Thus, $\lambda_{1}=\left(\begin{array}{c}n+1 \\ 2\end{array}\right)-2 n+1=\left(\begin{array}{c}n-1 \\ 2\end{array}\right), \lambda_{2}=1$, and $\lambda_{3}=2-n$, respectively.

\section{Singular Examples}

Let us consider the $n$-simplex in $\mathbb{R}^{n}$ with vertices $v_{0}, v_{1}, \ldots, v_{n}$ given as follows. The vertex $v_{0}$ has the first $n-2$ coordinates equal to $\left((n-1)^{1 / 2}+1\right) /\left(2^{1 / 2}(n-2)\right)$, while its last two coordinates are 0 . For $i=1,2, \ldots, n-2$, the vertex $v_{i}$ has $i$ th coordinate equal to $2^{-1 / 2}$ and all other coordinates 0 . These $n-1$ vertices form a regular $(n-2)$-simplex contained in $\mathbb{R}^{n-2} \subset \mathbb{R}^{n}$ with all side lengths 1 . Let $a=$ $\frac{1}{n-1} \sum_{i=0}^{n-2} v_{i}$ be its barycenter, and let $c:=\left\|v_{0}-a\right\|_{2}$ denote the distance from $a$ to the vertices $v_{i}$. A short calculation shows that $c^{2}=\frac{1}{2}-\frac{1}{2(n-1)}$. Now, let $v_{n-1}$ be obtained from $a$ by changing its last two coordinates to be real numbers $p$ and $q$ satisfying $p^{2}+q^{2}=1-c^{2}$. Similarly, let $v_{n}$ be obtained in the same way by choosing another pair $r, s$ of numbers satisfying $r^{2}+s^{2}=1-c^{2}$. This gives rise to an $n$-simplex whose all sides are equal to 1 except for the side $v_{n-1} v_{n}$ whose square length is $t:=(p-r)^{2}+(q-s)^{2}$. By fixing $t$, this simplex is determined up to congruence, and we denote it by $T(t)$. Observe that $t$ may take any value between 0 and $4\left(1-c^{2}\right)$, by selecting $p, q, r, s$ appropriately.

Next we observe that the volumes of the $(n-2)$-faces of $T(t)$ take only two values. If an $(n-2)$-face does not contain both $v_{n-1}$ and $v_{n}$, then it is a regular simplex, whose volume is independent of $t$. On the other hand, if an $(n-2)$-simplex contains $v_{n-1}$ and $v_{n}$, its volume $w=w(t)$ is uniquely determined by $t$. In fact, if we put the square distances in the Cayley-Menger determinant, we conclude that $w(t)^{2}$ is a quadratic polynomial in $t, w(t)^{2}=\alpha t^{2}+\beta t+\gamma$. If $t=0$, the volume is 0 , so $\gamma=0$. For $t=1$ we have the regular $(n-2)$-simplex, so $\alpha+\beta=\frac{n-1}{2^{n-2}((n-2) !)^{2}}$. Finally, using (1) (with the value of $n$ being replaced by $n-2$ ) and looking at the Cayley-Menger determinant expansion term with $t^{2}$, we conclude that 


$$
\alpha=-\frac{(-1)^{n-1}}{2^{n-2}((n-2) !)^{2}} \operatorname{det}\left(J_{n-2}-I_{n-2}\right),
$$

where $J_{n-2}$ is the all-1-matrix and $I_{n-2}$ is the identity matrix of order $n-2$. Since $\operatorname{det}\left(J_{n-2}-I_{n-2}\right)=(-1)^{n-3}(n-3)$, we conclude that $\alpha=-(n-3) 2^{2-n} /((n-2) !)^{2}$ and $\beta=(n-2) 2^{1-n} /((n-2) !)^{2}$. In particular,

$$
w(t)^{2}=\frac{1}{2^{n-2}((n-2) !)^{2}}\left((3-n) t^{2}+(2 n-4) t\right) .
$$

This function is symmetric around the point $t_{0}=\frac{n-2}{n-3}$. Consequently, the noncongruent $n$-simplices $T\left(t_{0}-x\right)$ and $T\left(t_{0}+x\right)$ have the same $(n-2)$-volumes of their $(n-2)$-faces for each admissible value of $x$, i.e., for $0<x<\frac{n-2-4 /(n-1)}{n-3}$. These examples thus show that Questions 1 and 2 have negative answers.

\section{Concluding Remarks}

One can ask the same question as above for other dimension-complementary volumes, i.e., about the volumes of the $(k-1)$-faces and the $(n-k)$-faces of an $n$ simplex, where $2 \leq k \leq n / 2$. If one would compare, similarly as in the case $k=2$ above, the dependence of $(n-k)$-volumes of an $(n-k)$-face $Q$ on the $(k-1)$ volumes of the $(k-1)$-faces $F \subset Q$, the corresponding "Jacobian" would again be a constant multiple of a symmetric matrix $M$, whose entries are indexed by the $k$-subsets of the set $R=\{1, \ldots, n+1\}$ (after the column indices pass to the complementary subsets), and

$$
M_{E, F}= \begin{cases}1, & \text { if the } E \cap F=\emptyset, \\ 0, & \text { otherwise. }\end{cases}
$$

The graph whose adjacency matrix is $M$ is known as the Kneser graph $K(n+1, k)$. Its eigenvalues can be computed using the methods from the theory of association schemes and can be found, for example, in [8, Sect. 9.4].

Theorem 8 Let $n$ and $k$ be integers, where $2 \leq k \leq n / 2$, and let $M$ be the matrix of order $\left(\begin{array}{c}n+1 \\ k\end{array}\right) \times\left(\begin{array}{c}n+1 \\ k\end{array}\right)$ whose entries are determined by (2). The eigenvalues of $M$ are the integers

$$
\lambda_{i}=(-1)^{i}\left(\begin{array}{c}
n-k-i+1 \\
k-i
\end{array}\right), \quad i=0,1, \ldots, k
$$

Since $2 \leq k \leq n / 2$, none of the eigenvalues in Theorem 8 are zero. This raises the question whether there is an analogy with Theorem 4 for $2 \leq k \leq n / 2$, between the collection of the $\left(\begin{array}{c}n+1 \\ k\end{array}\right)(n-k)$-dimensional volumes of the $(n-k)$-faces of an $n$-simplex and the collection of all $(k-1)$-dimensional volumes of its $(k-1)$-faces.

As a final remark, we would like to point out that our original approach to this problem [10] used results about divisors [6] (also known as equitable partitions [8]) combined with the representation theory of the symmetric group and the notion of Gelfand pairs as developed in [7]. 


\section{References}

1. Barrett, J.W.: First order Regge calculus. Class. Quantum Gravit. 11, 2723-2730 (1994)

2. Berger, M.: Geometry, vol. I. Springer, Berlin (1987)

3. Bianchi, E., Modesto, L.: The perturbative Regge-calculus regime of loop quantum gravity. Nucl. Phys. B 796, 581-621 (2008)

4. Biggs, N.: Algebraic Graph Theory, 2nd edn. Cambridge University Press, Cambridge (2001)

5. Blumenthal, L.M.: Theory and Applications of Distance Geometry. Clarendon Press, Oxford (1953)

6. Cvetković, D.M., Doob, M., Sachs, H.: Spectra of Graphs, 3rd edn. Johann Ambrosius Barth, Heidelberg (1995)

7. Diaconis, P.: Group Representations in Probability and Statistics. Institute of Mathematical Statistics Lecture Notes, vol. 11. Institute of Mathematical Statistics, Hayward (1988)

8. Godsil, C., Royle, G.: Algebraic Graph Theory. Graduate Texts in Mathematics, vol. 207. Springer, New York (2001)

9. McMullen, P.: Simplices with equiareal faces. Discrete Comput. Geom. 24, 397-411 (2000). The Branko Grünbaum birthday issue

10. Rivin, I.: Simplices and spectra of graphs arXiv:0803.1317v1

11. Smith, W.D.: Studies in computational geometry motivated by mesh generation. Ph.D. thesis, Princeton University (1989) 